\title{
Inferring Social Ties in Academic Networks Using Short-Range Wireless Communications
}

\author{
Igor Bilogrevic \\ LCA1, EPFL \\ Lausanne, Switzerland \\ Florent Lopez \\ Université Paul Sabatier/IRIT \\ Toulouse, France
}

\author{
Kévin Huguenin \\ LCA1, EPFL \\ Lausanne, Switzerland \\ Jean-Pierre Hubaux \\ LCA1, EPFL \\ Lausanne, Switzerland \\ Valtteri Niemi \\ University of Turku \\ Turku, Finland
}

\author{
Murtuza Jadliwala \\ Wichita State University \\ Wichita, USA \\ Philip Ginzboorg \\ Aalto University \\ Espoo, Finland
}

\begin{abstract}
WiFi base stations are increasingly deployed in both public spaces and private companies, and the increase in their density poses a significant threat to the privacy of connected users. Prior studies have provided evidence that it is possible to infer the social ties of users from their location and co-location traces but they lack one important component: the comparison of the inference accuracy between an internal attacker (e.g., a curious application running on a mobile device) and a realistic external eavesdropper in the same field trial. In this paper, we experimentally show that such an eavesdropper is able to infer the type of social relationships between mobile users better than an internal attacker. Moreover, our results indicate that by exploiting the underlying social community structure of mobile users, the accuracy of the inference attacks doubles. Based on our findings, we propose countermeasures to help users protect their privacy against eavesdroppers.
\end{abstract}

\section{Categories and Subject Descriptors}

C.2 [Computer-Communication Networks]: General; K.4.1 [Computers and Society]: Public Policy IssuesPrivacy

\section{Keywords}

Privacy; Social Networks; Mobile Networks; Inference

Permission to make digital or hard copies of all or part of this work for personal or classroom use is granted without fee provided that copies are not made or distributed for profit or commercial advantage and that copies bear this notice and the full citation on the first page. Copyrights for components of this work owned by others than the author(s) must be honored. Abstracting with credit is permitted. To copy otherwise, or republish, to post on servers or to redistribute to lists, requires prior specific permission and/or a fee. Request permissions from permissions@ acm.org. WPES'13, November 4, 2013, Berlin, Germany.

Copyright is held by the owner/author(s). Publication rights licensed to ACM. ACM 978-1-4503-2485-4/13/11 ...\$15.00.

http://dx.doi.org/10.1145/2517840.2517842.

\section{INTRODUCTION}

Innovative Internet mobile services and products, such as location-based services and information-sharing platforms, enable users to enjoy a multitude of applications to easily stay in touch, work, have fun and exchange data. Beside online services accessed through infrastructure-dependent communications (e.g., WiFi hotspots and cell towers), upcoming near-field [22] and peer-to-peer (P2P) technologies, such as Nokia Instant Community (NIC), and Qualcomm's AllJoin [25], allow for direct device-to-device connections, thus creating a new form of localized and context-aware interactions. For instance, Apple's AirDrop allows users to exchange files between mobile devices by creating an ad-hoc WiFi network. All in all, the amount of data exchanged wirelessly by mobile devices, be it in an infrastructure or $\mathrm{P} 2 \mathrm{P}$ ad-hoc mode, has consistently increased over the past few years.

The information that can be inferred only by observing the data exchange patterns, users' location and co-location traces is of great importance and very sensitive: Home/work locations [18], activities [21], and social networks [9] are often data that people would prefer not to reveal publicly. Users' social ties are no exception either, as several studies on ubiquitous computing have shown that location and co-location traces alone can reveal the presence and type of social relationships among people $[11,12]$. Most of the existing works that evaluate the effectiveness of the inference of social ties from location and proximity data are based on a single source of information: either proximity logs stored on the mobile devices or WiFi/cellular connection data obtained from the network operators. Although the existing results are significant and necessary for an initial attempt at tackling the issue of social relationship inference, they lack an important characteristic: data-source diversity for the same experimental settings. In other words, they lack the simultaneous access to proximity data present on both the mobile devices and in the operators' logs. Having access to these two data sets is paramount, as this enables us to evaluate and compare the accuracy in inferring the social relationships between an internal attacker, who has access 
to the data on the device, and an external eavesdropper who does not.

In this paper, we address the problem of social relationships inference by carrying a comparative analysis of the exposure of such relationships and encounters in a deployed, peer-to-peer and infrastructure-based wireless network. We gathered communication and proximity information from 80 participants carrying smartphones during a four-month experiment; Thus our study is the first to possess data-source diversity for the same experimental setting: We collected complementary encounter information both from the smartphones and from the WiFi sniffing network, comprised of a set of access points (APs) passively intercepting communications between the participants. These two sources reflect accurately the information that can be gathered by (i) a curious application (or rogue firmware) running on a smartphone or an experimenter and (ii) a network infrastructure owner such as a company which seeks to infer the social relationships between its employees or a network of compromised (community) access points; both of them represent realistic and practical adversarial scenarios. By leveraging on such complementary data, we design a statistical inference framework to evaluate the accuracy of the inference of social relationships in localized and pervasive networks. This evaluation provides empirical evidence about the difference between the adversary's accuracy and that of a curious application in determining the type of social relationships between people. More specifically, our contribution is twofold:

- We provide the first analysis, to the best of our knowledge, of the exposure of social relationships, based on proximity data, in a deployed adversarial WiFi network with data-source diversity.

- We design a statistical inference framework for relationship classification and use it to evaluate the success of the owner of the WiFi sniffing stations in inferring the type of social relationships between users and compare it against a curious application.

Our experimental results show that the infrastructure operator is able to infer the relationships between users better than a curious application that uses on-device proximity data. This is partially due to the fact that the external adversary has location information about the encounters between users. In addition, when exploiting the social community structure, the inference accuracy of the attacker is significantly higher. To put our results in perspective, we compare the strength of the considered adversary, in terms of the density of sniffing stations, to some existing hotspot networks.

The remainder of the paper is organized as follows. In Section 2, we introduce the experimental setup and system architecture. In Section 3 we detail the characterization of the social interactions and communities. We describe our relationship inference framework in Section 4 and its application to the experimental data. In Section 5, we present the results of the inference framework and their implications on the privacy of users' social relationships. We also discuss the limitations and perspective of this work, as well as countermeasures to help users protecting their privacy. Finally, we survey the related work in Section 6 and we conclude the paper in Section 7.

\section{SYSTEM ARCHITECTURE}

In this section, we describe the methodology and experimental setup of our field experiment, along with the network model and the adversarial model.

\subsection{Experiment Setup}

During the spring of 2011 (from the beginning of March to the end of June 2011, we conducted a large-scale field experiment with 80 participants on a university campus, during which we collected encounter and proximity data. Similarly to previous data collection campaigns [11,17], we configured Nokia N900 smartphones and distributed them to the participants, sampling from a coherent population of students $(96 \%)$ and instructors (4\%), $80 \%$ of the participants being males. The participants answered survey questionnaires at the beginning and in the middle of the experiment, and they were individually interviewed at the end of the experiment. The relevant answers to such questionnaires are used in order to construct the ground truth of user relationships. The complete description of the goals and the methodology of the trial, the questionnaires and the interviews is reported in [1]. The experiment was conducted with Nokia and the participants signed a consent form prepared by Nokia and approved by their legal and ethical department.

\subsection{Network Model}

The smartphones were configured with both standard infrastructure-based communications, such as cellular and WiFi, and a WiFi-based P2P technology called Nokia Instant Community (NIC). Users could connect to standard Internet services by using the WLAN or cellular interface of the device. Also, in order for the participants to exchange information with their physical neighbors in a P2P fashion (Figure 1a), the phones were equipped with the NIC wireless P2P messaging platform that allows for localized data exchange and increases the co-located devices' contextawareness. In addition, several campus and course-related applications were developed in order to encourage the use of the devices throughout the duration of the experiment. To enhance the context-awareness of the pre-installed applications, the devices periodically sent beacon messages and ran background services that collected and stored in the local memory, at regular intervals of [1-30] seconds, information such as the list of neighbors' MAC addresses, the associated received signal strength indicator (RSSI) and the timestamp. Each participant was identified by a fixed random ID assigned at the beginning of the trial, and this ID remained unchanged. Considering dynamically changing user IDs, such as temporary pseudonyms in mix-zones [4], is outside of the scope of this paper. Note that, as of today, most smartphones do not offer easy ways to change their MAC addresses and even when they do, most users never change them manually. And using changing pseudonyms has a negative effect on the usability of the communication features of the device and brings only limited improvement. Whenever a participant's device connected to the Internet, the new encounter logs were uploaded on a centralized database storing all device logs. To preserve users' anonymity, we removed all personal identifier information (such as the mapping between MAC address, IMEI, and participant name) from the database. In order to construct the ground truth for our experiments, we kept only the information about (i) the ran- 


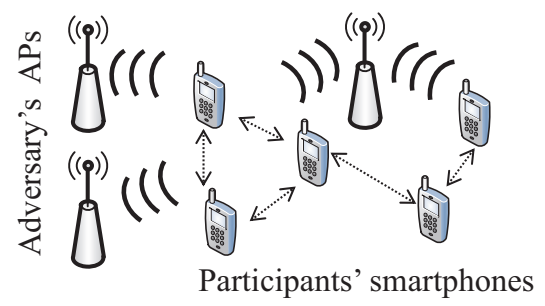

(a) Trial network architecture.

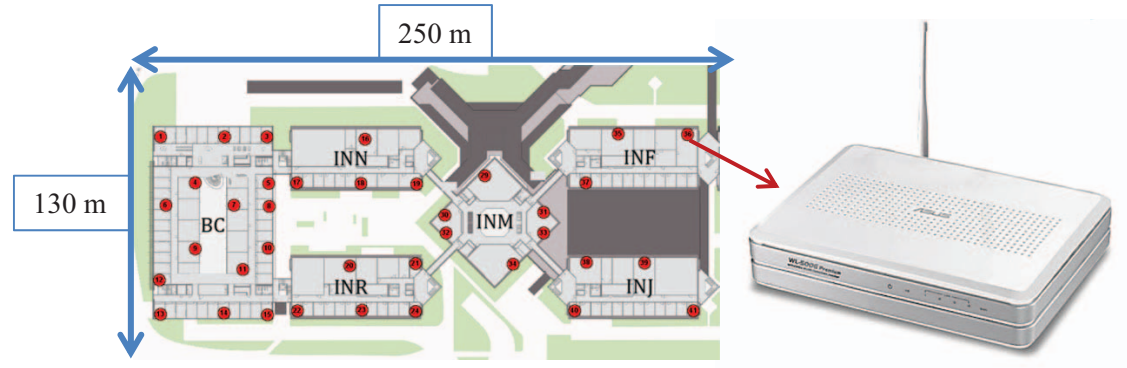

(b) Map of the deployed wireless network of 37 APs controlled by the adversary.

Figure 1: Trial setup and deployed eavesdropping network controlled by the adversary.

dom user IDs, (ii) their self-reported relationships and (iii) the academic registration data (mapped to the user IDs).

\subsection{Adversarial Model}

We implemented a practical adversary who monitored a fixed area by using a limited number of wireless sniffing stations comprised of either (i) dedicated WiFi APs deployed directly by the adversary or (ii) existing APs compromised by a malware $[19,27]$, which is a realistic threat to which most of the reconfigurable, current-generation WiFi APs are exposed to. Specifically, the adversary had access to a deployed wireless mesh network of $37 \mathrm{WiFi}$ APs (Asus WL-500gP running OpenWRT Linux) in a specific region of the campus, covering one level of six interconnected buildings that have a very high user (student) density (Figure 1b). The relative strength of the adversary in terms of the density of APs, is studied by conducting a comparison with a deployed hotspot networks. The coverage area included the classrooms in which two popular courses that the students attended took place. Each AP passively eavesdropped on the NIC packets generated by the participants' smartphones, and it periodically uploaded the eavesdropped data to a server, populating a unified log database. The APs were set in promiscuous mode on the fixed channel used by NIC. Moreover, the adversary did not have access to any information stored on the mobile devices, and we assume that the devices were honest (i.e., not colluding with the adversary).

In order to infer the social relationships, the adversary collected the 3-tuple (Timestamp, Source MAC, RSSI) from the sniffed NIC messages. Note that at no time did the participants connect to the Internet through the adversarial APs, that were used only for passively sniffing the NIC packets. Due to the localized nature of the NIC message exchanges, they can be captured only by a local adversary. Such data arguably leaks an amount of personal information lower than the data that flows through the infrastructure. Because application-layer encryption was sometimes used to protect the message confidentiality, we assume that the adversary did not have access to such data. All adversarial knowledge is built from the analysis of the NIC data, passively collected by the sniffing APs.

\section{SOCIAL INTERACTIONS AND COM- MUNITIES}

In this section, we describe the methodology used to model the user interactions during the experiment. The social graph representing the user interaction is used to infer the community structure, which is subsequently used to refine the social relationships inference mechanism.

In society, people tend to organize themselves in social groups or communities, such as families, colleagues, and friends, where members usually have similarity traits with other members stronger than with non-members [15]. From a graph-theoretic perspective, people and their relationships can be represented by an undirected weighted graph $G=(V, E, W)$, where the vertex set $V$ corresponds to people, the edge set $E$ expresses the existence of a relationship between people, and the weight function $W: E \rightarrow \mathbb{R}^{+}$ quantifies the intensity of such relationships. In their simplest form, communities can be represented as subgraphs $\left\{C_{i}=\left(V_{i}, E_{i}, W\right)\right\}_{i=1}^{M}$, where $C_{i} \subseteq G$ and $M$ is the number of communities. As people can be members of several communities, different community subgraphs can share vertices.

\subsection{Experimental Data}

In our experimental data, we have two sources of proximity information: (i) the internal data, constituted by local device logs collected by the mobile devices themselves (the devices of all the participants have been compromised by the internal adversary, e.g., through a rogue firmaware update) and containing encounter data (list of neighbors' MAC addresses, the timestamps and the RSSI values of received packets), and (ii) the adversarial data, containing the headers of the sniffed packets (sent by the mobile devices), which include the timestamps and RSSI values of received packets at the sniffing APs, as well as the device ID of the sender.

We use these two data sources to formulate the 'strength' or intensity of the social relationships between users and to define the weights of the edges that connect the respective vertices in the social graph $G=(V, E, W)$. From the local device logs, we can directly obtain the device-to-device proximity information because the recorded RSSI values on the receiving devices are correlated with the real distances to the sending devices. However, this is not the case for the RSSI values recorded by the adversarial network, as they depend on the distance between the sending device and the receiving sniffing station. Therefore, the adversary needs to derive the device-to-device proximity information from the device-toAP RSSI values: the adversary first estimates the position of a device and then computes the device-to-device proximity information in order to determine the weights between vertices of the graph. To this end, we used a localization algorithm based on trilateration; it determines the estimated position of a mobile device that sent a packet based on the RSSI at all sniffing stations that overhear the packet. 


\section{Relationship Labels}

In addition to the strength of social relationships, we also characterize the type of relationship between users. More specifically, we consider three labels: (i) friends, (ii) classmates and (iii) others. ${ }^{1}$ The possibility of associating multiple labels to a single relationship is crucial in social networks, as people can be simultaneously classmates and friends. To construct the ground truth data, each relationship is assigned one (or more) labels based on (i) the participants' answers to the survey questionnaires (for friends) and (ii) the database of academic course registration provided by the university (for classmates). For instance, if two participants followed the same class during the experiment, their relationship was categorized as classmates; furthermore, if one of the same participants also listed the other as friends in the questionnaires, their relationship was categorized as both classmates and friends. We note here that for the friendship label, due to the lower number of declared friendships, we considered asymmetric relationships as symmetric because of the subjective nature of the friendship relationship that could be perceived by one person and not by the other. From our ground truth data, we observed that $25 \%$ of all the 3,160 possible relationships were tagged as classmates and $2 \%$ as friends. We tagged the remaining as others.

We choose these types of relationships for two reasons. First, we followed the approach of [7] and selected the types of relationship relevant to our context. For instance, as our population was comprised almost exclusively of students on a university campus, we considered the two relationship types aforementioned, as well as the estimated user location. For a more diverse on-campus population, including researchers and instructors, we would have had to consider the role of the hierarchy as well. Second, it was possible for us to collect the ground truth for these two types of relationships.

In our proximity network, we consider three distinct weight functions $\left\{w_{i, j}^{(d)}\right\}_{d=1}^{3}$ for the edges between vertices $i, j \in V$ to quantify the intensity of their social interactions. The three weight functions [5], progressively take into account the proximity, the intensity, and the aging/recency of the relationships between users. Moreover, to investigate the effect of community detection on the accuracy of the inference attack, we apply the well-suited CPM method [24] to extract community information from the social graph $G$. In the Evaluation section we present the results of the attacker's success for each of the three weight functions, with and without community detection.

\section{RELATIONSHIP INFERENCE FRAME- WORK}

In this section, we introduce our relationship inference framework by which we evaluate the accuracy of the attacker's reconstruction of users' social relationships.

\subsection{Relationship Characterization}

The relationships between two users are characterized by their interactions. To characterize and classify relationships, we first formalize the notion of encounter: it captures a

\footnotetext{
${ }^{1}$ Friends and classmate relationships can be more easily recognized based on just on-campus encounter data. More complex relationships, such as "lovers" and "family", require additional contextual data which our dataset does not include.
}

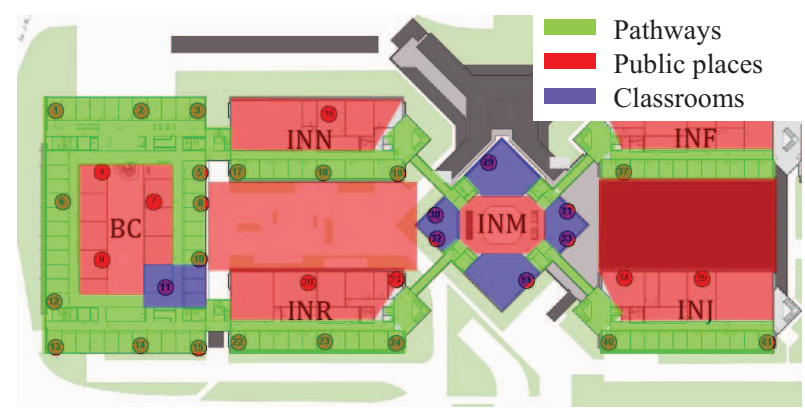

Figure 2: Partition of the map (in which the experiment was conducted) into three types of areas: pathways, public places, and classrooms.

significant interaction between two users. A relationship is defined by a set of encounters.

\section{Encounter Detection}

We define an encounter between any two users as a continuous time interval that meets a threshold, both on its duration and on the proximity of the two involved users. Typical values of these thresholds are 5 minutes and a 20 meters, respectively. The proximity between users is computed in two ways, according to the source of the data. From the internal device logs, we use the RSSI values of messages exchanged between users (in a P2P fashion) in order to determine their distance, whereas from the adversarial sniffed data, the proximity is determined by computing the Euclidian distance between the estimated location coordinates of each user, based on device-to-AP RSSI values.

A number of features are extracted from the internal/adversarial logs, either at the granularity of an encounter or of a relationship. For instance, we extract the duration of each single encounter and the total number of encounters between the two users for each relationship. The extracted features are then fed to the classification algorithm. In order for the adversary to accurately classify the relationships based on specific features, these features must vary significantly from a category (such as friends) to another. Typical discriminating features include encounter duration, the proximity of users during encounters, interencounter time, and the number and periodicity of encounters $[28]$.

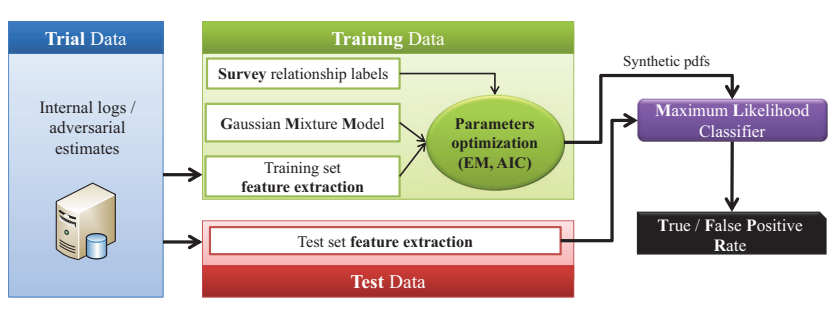

Figure 3: Overview of the inference framework.

\subsection{Relationship Classification}

In order to select the set of possible relationship classes, we use the ground-truth collected through (i) survey questionnaires at the beginning of the trial and (ii) the official 
university course-registration database. According to the answers of the participants and the university register, we selected three classes of pairwise relationships between users, i.e., friends, classmates, and others. We then designed an inference framework (Figure 3) based on the features extracted from the internal/adversarial data.

The classification algorithm operates as follows. First, we extract the features of a small subset of the encounters and relationships, namely a training set. From the survey and administrative records, we know the category of each encounter and relationship in the training set. Then, for each of the three categories of relationships, we compute the experimental probability density function (pdf) of the various features and plot the corresponding histograms. We fit each experimental probability density function with an appropriate parametric model, the Gaussian Mixture Model (GMM) [11], and determine the parameters according to two optimization criteria. The obtained synthetic pdfs are subsequently used to classify the encounters of the rest of the data set, i.e., the test set. Based on the synthetic pdfs, for each encounter and for each category of relationships, the likelihood that the encounter belongs to this category is computed from the values of its features. The likelihood that a relationship belongs to a given category is computed by taking into account the different features of the relationship and those of its encounters.

\subsubsection{Encounter Location Classification}

In our work, the location where encounters take place is a key feature of relationships. This information is only available in the adversarial data and is computed from the users' locations estimated by the adversary. More specifically, the region of the experiment covered by the adversarial network is partitioned into different areas classified in three types: pathways, public spaces and classrooms (Figure 2). We expect such a classification to be discriminating, with respect to the types of relationships the adversary wants to infer. For instance, classmates are expected to experience frequent encounters mostly in classrooms, whereas friends might hangout more frequently in public spaces and pathways. Because users can move during a given encounter, for each encounter, we compute the proportion of the time spent in each type of location. In this way, we can capture the fact that an encounter that started in a pathway continues through public spaces.

\subsubsection{Feature Extraction}

We detect encounters based on interaction duration and proximity between users. With the internal data, we assume that an encounter occurred if at least two messages are exchanged within an interval of 5 minutes and the average RSSI of the exchanged messages is greater than $-80 \mathrm{dBm}$ (to account for radio noise, interference and fading), which corresponds to a distance of at most 18 meters. These threshold values have been determined empirically. The encounter spans from the first exchanged message to the last. For example, if two users exchange messages, all with RSSI values of $-50 \mathrm{dBm}$, at times $1,3,7$, and 15 minutes, an encounter is detected and it spans from 1 to 7 minutes (the last message arrives too late to extend the encounter duration). With the adversarial data, we first need to estimate the users' locations. In order to reduce the noise on the users' estimated locations, we divide the trial timeline in small sub-intervals of 30 seconds and we average user locations over each subinterval. First, we consider that an encounter occurs if at least two messages are exchanged within an interval of 5 minutes and the distance between the two users' locations is consistently lower than 5 meters (i.e., lower than 5 meters in each sub-interval between the times at which the messages were exchanged). To compare the results with the internal data, we also considered distance thresholds of 10, 20, 30, and 40 meters, corresponding to RSSI values of -68, -82, -91, and $-97 \mathrm{dBm}$, respectively (according to the Haka-Okumura radio propagation model [8]).

At the granularity of an encounter, we extract the following features: (i) the encounter duration (the time elapsed between the first and last message exchanged during the encounter), (ii) the inter-encounter time (the time elapsed since the end of the previous encounter and the beginning of the current encounter), and (iii) the average encounter RSSI value (the average of the RSSI values of the messages exchanged during the encounter). For the adversarial data, we further consider (iv) the encounter location, characterized by the fraction of the encounter time that takes place at each type of location, such as pathways, public places and classrooms. The location of an encounter is defined as the midpoint of the two users. At the granularity of a relationship, we consider an additional feature: the total number of encounters over the whole trial.

\subsubsection{Supervised Learning}

In order to train our inference framework, we use a random subset (the training set) of all the relationships, which account for approximately $30 \%$ of the whole data set. We divide the relationships in the training set into three categories, i.e., friends, classmates, and others according to the labels obtained from the surveys and the university database. Because two users can simultaneously be friends and classmates, a relationship can belong to both categories. Note that an alternative way to proceed would be to divide the training set into four categories: friends, classmates, others, and friends and classmates. However, as in our data set the "friends and classmates" category does not contain enough relationships to perform a proper training, we consider only the first three categories: friends, classmates, others. For each category, and for each feature, we compute the experimental distribution of the feature for all the relationships in the category, by means of histograms composed of 100 bins. We denote by $\tilde{f}_{\text {cat }}^{\text {feat }}$, cat $\in\{$ friends $(f)$, classmates $(c)$, others $(o)\}$ and feat $\in$ \{encounter duration (ed), interencounter time (iet), average RSSI (rssi), and number of encounters (ne)\} (and encounter location (el) for the adversarial data), the functions corresponding to the histograms. For example, $\tilde{f}_{\mathrm{f}}^{\text {ed }}(x)$ gives the proportion, in the training set, of encounters between friends that last between $x$ and $x+\delta x$ minutes. These functions can be thought of as indicators of the likelihood that a given encounter belongs to a given category, based on the value of one of its features, and will be used as such by our classification algorithm.

Intuitively, it can be expected that the pairs of users whose relationships are classified as friends and classmates would experience more frequent encounters than users belonging to the category others. Similarly, classmates are expected to meet according to a fixed pattern (e.g., every Monday for a particular class), whereas on-campus encounters between friends are not expected to follow a fixed pattern. This intu- 
ition is confirmed by our observation of the data, as shown in Figures 4 and 6 . The former depicts the experimental distribution of two features: the encounter duration (at the granularity of an encounter) and the number encounters (at the granularity of a relationship), whereas the latter shows the location feature. It can be observed that, as expected, these features discriminate the three categories of relationships, therefore we foresee a high potential for classification. For instance, friends meet more than classmates and others and classmates meet for longer times (classes). Also, classmates meet mostly in classrooms. Note that the aforementioned intuitions are given for the sake of information, the inference algorithm is based on the rigorous techniques that we describe.
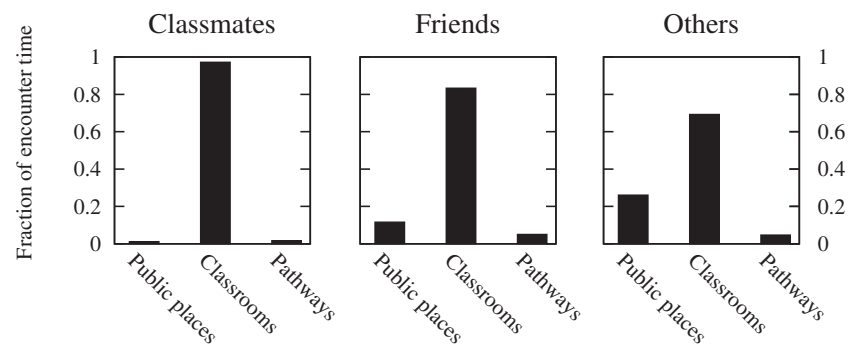

Figure 4: Encounter location feature for the adversarial data (distance threshold of $\mathbf{4 0}$ meters).

As it can be observed in Figure 6, the experimental distributions are noisy and thus cannot be used as is for classification purposes: For instance, we can observe in the histograms that there are no relationships between friends composed of exactly 35 encounters in our training set. Therefore, if the histogram of the number of encounters is used as is to determine the likelihood of two users being friends, two users who meet 35 times during the trial would be assigned a null likelihood for the friends category. For this reason, we fit the experimental histograms with parametric pdfs, namely multi-dimensional Gaussian mixture models of the form:

$$
p(\mathbf{x} ; \theta)=\sum_{k=1}^{K} \pi_{k} \frac{1}{\sqrt{\left|\Sigma_{k}\right|}(2 \pi)^{d / 2}} e^{-\frac{1}{2}\left(\mathbf{x}-\mu_{k}\right)^{\mathrm{T}} \Sigma_{k}^{-1}\left(\mathbf{x}-\boldsymbol{\mu}_{k}\right)}
$$

where $\sum_{k} \pi_{k}=1$ and $d$ is the dimension of the feature vector. The set of parameters is denoted by $\theta=\left(\left(\pi_{1}, \boldsymbol{\mu}_{1}, \Sigma_{1}\right)\right.$, $\left.\ldots,\left(\pi_{K}, \boldsymbol{\mu}_{K}, \Sigma_{K}\right)\right)$, and $\mathbf{x}$ is the value of a feature. We use one-dimensional GMMs for scalar features, e.g, encounter duration, and multi-dimensional GMMs for the encounter location (fractions of time spent in pathways, classrooms, and public places).

The number $K$ of Gaussian components, and their respective weights $\left(\pi_{k}\right)$, means $\left(\boldsymbol{\mu}_{k}\right)$, and covariance matrices $\left(\Sigma_{k}\right)$ are free parameters and must be chosen to best fit the experimental distributions. For a given value of $K$, we determine the other free parameters of the Gaussian components through expectation maximization (EM). We choose the values of the parameters that maximize the likelihood that the $n$ values $\left\{\mathbf{x}_{i}\right\}_{i=1 . . n}$ of the features (observed in the training set) have been drawn from a Gaussian mixture with these parameters. The likelihood function is given by

$$
\ell\left(\theta ; \mathbf{x}_{1}, \ldots \mathbf{x}_{n}\right)=\prod_{i=1}^{n} p\left(\mathbf{x}_{i} ; \theta\right)
$$

and the estimated value of the parameters is $\hat{\theta}=\arg \max _{\theta}$ $\ell\left(\theta ; \mathbf{x}_{1}, \ldots \mathbf{x}_{n}\right)$. We repeat this estimation step for several values of $K$, and we subsequently determine the most appropriate value of $K$ according to the Akaike information criterion [2]: $\mathrm{AIC}=2 k^{\prime}-2 \ln \ell(\theta)$, where $k^{\prime}$ is the number of parameters of the GMM.

The learning phase produces a likelihood function $\ell_{\mathrm{cat}}^{\mathrm{feat}}$ for each category cat $\in\{f, c, o\}$ and for each feature feat $\in$ $\{$ ed, iet, rssi, ne\} (and el for the adversarial data).

\subsubsection{Relationship Classifier}

Our classification algorithm is based on likelihood maximization and operates in two steps. We first determine if the two users are actually involved in a relationship (friends or classmates) or not (others) based on the likelihood of a relationship, with respect to each category. This likelihood is computed from the value of the features and from the synthetic distribution obtained from the training set. If users are believed to have a relationship, we refine the classification by discriminating between friends and classmates.

More specifically, the algorithm operates as follows (see Figure 5 for illustration). If the likelihood is maximized for the others category, the relationship is classified as others and the classification ends. If it is maximized for the friends category, the relationship is assigned the friends label and a second step is performed to decide whether the classmates label is assigned as well. This second step is based on a threshold: If the likelihood of the relationship for the category classmates is higher than the likelihood of friends multiplied by a factor $\gamma \in[0,1]$, the relationship is assigned the classmates label as well. The same process applies in the case the likelihood is maximized for the classmates category. The classification process can be summarized by the decision tree depicted in Figure 5.

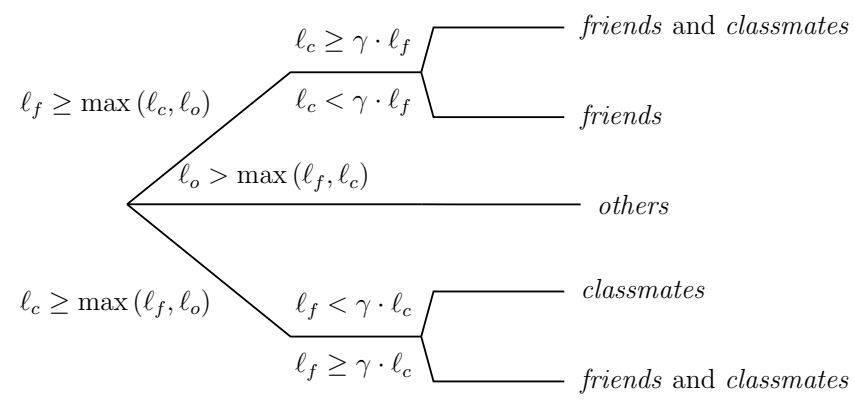

Figure 5: Decision tree used by the classifier.

The likelihood $\ell_{\text {cat }}$ of a relationship, with respect to each category cat, is given by

$$
\begin{array}{r}
\ell_{\mathrm{cat}}(r)=\left(\prod_{e \in r} \ell_{\mathrm{cat}}^{\mathrm{ed}}(\mathrm{ed}(\mathrm{e})) \cdot \ell_{\mathrm{cat}}^{\mathrm{iet}}(\mathrm{iet}(\mathrm{e})) \cdot \ell_{\mathrm{cat}}^{\mathrm{rssi}}(\mathrm{rssi}(\mathrm{e}))\right) \\
\times \ell_{\mathrm{cat}}(\mathrm{ne}(\mathrm{r})),
\end{array}
$$

where $m \in r$ denotes the encounters between the two users concerned by the relationship $r$. For the adversarial data, 

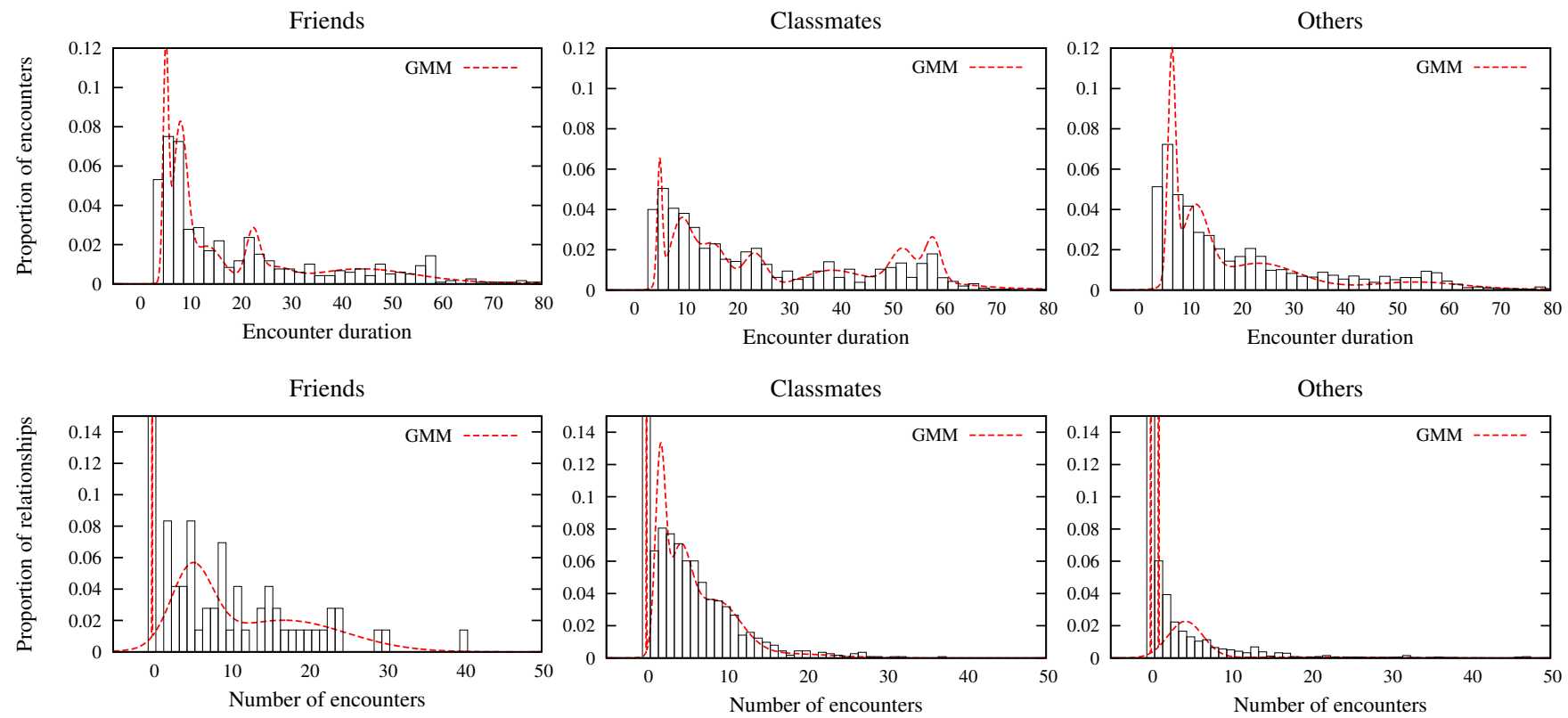

Figure 6: Experimental distributions (histograms) and fitted GMM pdfs (dashed lines) of two features extracted from the adversarial data (distance threshold of 40 meters): encounter duration and number encounters.

the factor $\ell_{\mathrm{el}}(\mathrm{el}(e))$ must be inserted in the product in Equation (3) to take location into account.

\section{RESULTS}

In this section, we present the performance results of our relationship classification inference framework. First, we describe the metrics used to evaluate the inference accuracy of the adversary, then we discuss the performance of the classifier and the effect on the users' relationship privacy. Finally, based on our results, we discuss possible countermeasures to mitigate the success of the adversary in inferring social relationships. We evaluate his success by using both pairwiseonly and community-enhanced proximity information.

\subsection{Pairwise Relationship Inference}

The performance of a classifier is usually evaluated in terms of its true positive rate (TPR) and false positive rate (FPR). Hereafter, we present the accuracy of the adversary's relationship inference on our data set, where the adversary considers only the estimated pairwise proximity information between users, i.e., without taking into account the underlying community structure. Figure 7 a shows the TPR and FPR for the three classes for different values of the distance threshold.

Internal Proximity Data. By using the internal proximity information between users, the performance of our inference framework is, in general, satisfactory, given the limited amount of training samples (30\% of the total). This means that we have a high TPR and a moderate FPR. For the class friends, we observe the best TPR (84\%) and a moderate FPR (27\%), which means that the actual proximity data and the encounter features we selected for the classification are quite discriminating for this class. Classmates and other relationship classes have a TPR/FPR of
$56 \% / 18 \%$ and $37 \% / 13 \%$ respectively, which are lower than for friends. As a result, the actual proximity data works well for the friends and classmates relationships inference, whereas it has a more limited success in classifying other types of relationships. This is not surprising, as proximity information, without taking into account the actual location of the interaction, is intuitively tied to inferring close and periodic interactions more than sporadic encounters.

Adversarial (Estimated) Proximity Data. First, at a lower encounter-distance threshold (5-10 meters), the adversary has a very high TPR rate for classmates and friends $(79 \%$ and $74 \%$ respectively) while it suffers from an elevated FPR as well ( $53 \%$ for friends). The accuracy for others is relatively low at $28 \%$, meaning that the prediction suffers from a significant number of false positives that negatively affect the successful recognition of friends and classmates. Whereas, for larger encounter-distance thresholds (20-40 meters), the accuracy for others doubles and is consistently larger than for the friends.

Internal vs. Adversarial. For low encounter-distance thresholds, on one hand the external adversary has a similar or higher accuracy in correctly inferring the relationships of participants compared to the case of the internal adversary (i.e., using the actual proximity data). On the other hand, the FPR is also substantially higher, which renders the relationship prediction much less reliable at low distance thresholds. As the adversary can estimate, in addition to users' proximity, some contextual information (location of the encounter), it is easier for him to infer the type of relationship given this additional feature, but it is also easier to wrongly include other types of relationships in the friends class.

When increasing the distance threshold (20-40 meters), we can see that the success in inferring friendships from external data decreases by at least $50 \%$ when compared to 


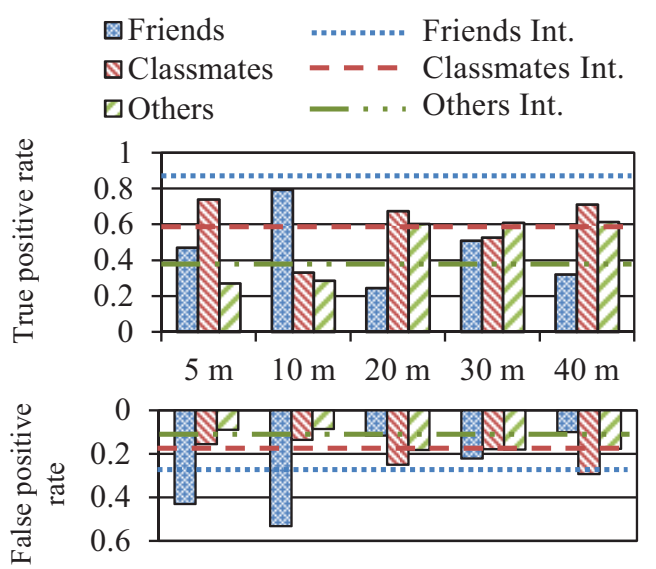

(a) Pairwise-based classifier accuracy.

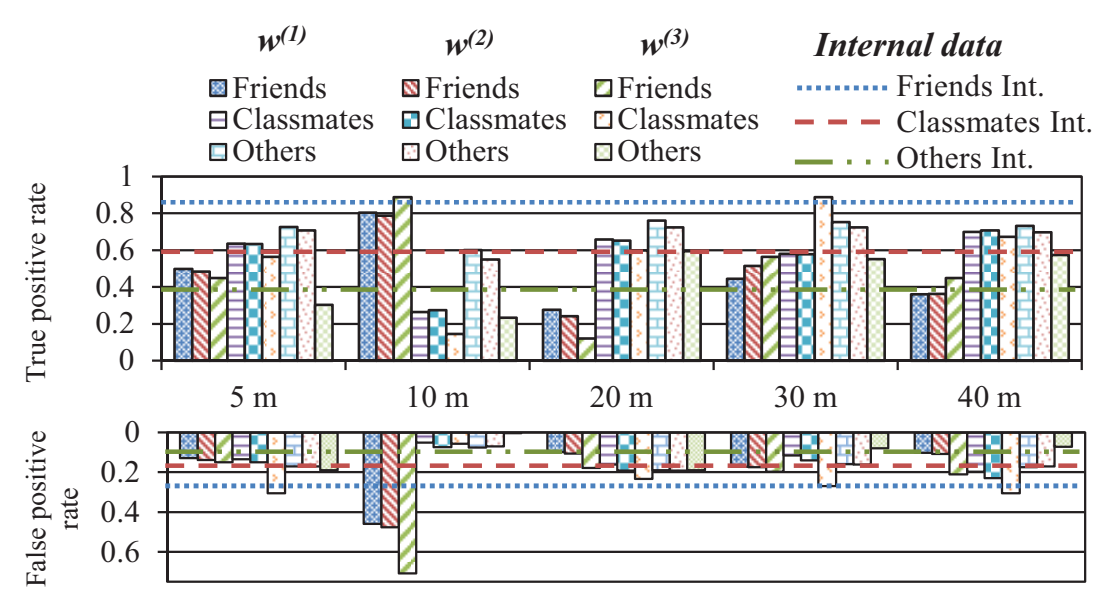

(b) Community-enhanced classifier accuracy.

Figure 7: Accuracy of the external adversary in inferring social relationships. The horizontal lines correspond to the accuracy of the classifier when the internal (local device) proximity data is used.

the case where internal data is used. Meanwhile, there is a two-fold increase in TPR for the others category. In other words, the larger the allowed distance between users is, the better the inference accuracy for the others category. And the classmates performance stays at comparable levels for the different distance thresholds.

Overall, we observe that the increase of the encounterdistance threshold for the adversary is highly beneficial for the detection of non-friendships. By adjusting such a threshold based on the target relationship class, the external adversary obtains an inference performance comparable to that of the internal adversary who uses the actual proximity information available on the devices.

\subsection{Community-Enhanced Relationship In- ference Results}

Hereafter, we describe the performance of the relationship inference framework when the adversary relies on the underlying structure of user communities. This pre-processing step will enable us to observe the effect of such information on the accuracy of the adversary's inference compared to the previous case without community information. The results are shown in Figure 7b.

Internal vs. Adversarial. For low-distance threshold values ( 5 meters), we see that the external adversary has a TPR comparable to the case where no community information is extracted (for most weight functions). However, the FPR values are significantly different: The false-positive rate for friends and classmates relationships is three to four times lower when using community structure than when not. When inferring the others class of relationships, the TPR is three times better than the community-less counterpart, with only a comparable FPR. These results indicate that by using the community structure of social relationships among users, the adversary is able to significantly increase his performance (both in terms of TPR and FPR) across all relationship types, especially in terms of false positives. With respect to the weight functions we defined for the CPM community detection algorithm, we can see that the first two functions $w^{(1)}$ and $w^{(2)}$ (see [5] for a detailed definition and formulation of the weight functions) have a slightly better performance for friend and classmate relationships and are twice as good for the others class. This suggests that by modeling the interactions of non-friends or classmates with memory-less weight functions, the detected community structure is better suited for distinguishing between such types of relationship, as they did not exhibit most of the periodic and close interactions during our experiment as friends or classmates.

\subsection{Discussion}

The results we have presented so far show that, even without any additional pre-processing by the adversary, the estimated location and proximity among mobile users is often enough to make accurate predictions about the kind of social relationship between any two users (Figure 7a). Even if the users rely on encryption for their communications, the considered external adversary can distinguish users as friends, classmates or neither by capturing their identifiers and by correlating them over time and space.

Even more striking is the fact that by adding an extra step in the inference process, both the accuracy (TPR) and reliability (FPR) are improved significantly. In particular, the dynamic social community structure of people and their behavior over time leak a substantial amount of additional private information to the adversary. By pre-processing the proximity data and detecting communities, in most cases the adversary is able to double his performance when inferring the types of relationships between users inside communities, much to the detriment of the privacy of users and their communities. This suggests that even a simplistic characterization of human encounters, based on memory-less and time-independent functions, GPS-less data and standard trilateration positioning algorithms, is enough to enable a wireless infrastructure owner to successfully detect communities and to determine the type of relationships among users inside each community.

Finally, by transposing our results to existing wireless networks, we hypothesize that similar findings can be obtained by self-interested or curious third-parties, such as commercial service providers, cellular network operators, 
WiFi network operators (commercial, companies, instituions). The increasing deployment of low-range cellular base stations [13] and the increased availability of public WiFi hotspots [14] severely undermine people's ability to resist or opt-out from unwanted scrutiny.

\subsection{Countermeasures and Limitations}

Countermeasures. A solution for limiting the success of the inference attack, which is specific to the threat presented in this paper, consists in reducing the information available to the adversary and his confidence in this information. To achieve this, technical solutions include the use of changing pseudonyms as wireless identifiers and sending dummy messages. With pseudonyms, users can change their MAC address every day while off-campus or, upon encounters, collectively interrupt all communications for a certain time and reconnect with fresh random pseudonyms (i.e., mix-zones [4]). The use of pseudonyms makes it difficult for the adversary to link and track identities of users over time. It reduces the learning period to a few hours instead of a the whole trial timespan, therefore decreasing the success of the inference. However, such a mechanism has a negative effect on the usability of the communication features, e.g., Bluetooth pairing and MAC filtering. Another technical countermeasure is to dynamically change the transmission power to confuse RSSI-based triangulation and limit the eavesdropping possibilities. However this comes at the cost of decreased networking performances. Finally, injecting dummy messages would artificially increase the intensity of the encounters, thus, biasing the results of the inference.

Limitations. The results and discussions presented in this study are based on a large-scale experiment conducted on a university campus, where most of the participants are students. The characteristics of such a population are rather homogeneous, with respect to the utilization of technology and the age. As in several other university-based field experiments, the results of our study might apply - to a lower extent - to scenarios involving a more diverse population with respect to education, age, gender and technology usage habits. In order to mitigate this, as part of our future work we would like to extend our experiment to a more diverse population, and therefore to assess the effectiveness of the inference attacks in a more mixed environment. Finally, as off-campus interactions are more insightful with respect to social relationships - for instance, the larger variety of location types (e.g., bars, cinemas and residential areas instead of just public places and classrooms) - we expect a greatly improved performance of the inference when considering off-campus interactions as well. This would enable us to consider fine-grained social relationships, such as close friends and relatives.

Strength of the Adversary. Regarding the relative strength of the adversary considered in the paper, with respect to the number of WiFi APs, we studied the density of three deployed WiFi AP networks for comparison. First, we looked at the FreeWifi [16] and SFRWifi [26] hotspots networks that consist of the wireless modems and set-top boxes of the subscribers of two major ISPs (Free and SFR respectively, which have $\sim 5 \mathrm{M}$ subscribers each for a population of $65 \mathrm{M}$ ) operating in France. Such networks of hotspots constitute concrete and illustrative examples of the adversary considered in this paper as they are operated by a single entity, i.e., the ISP. In particular, the ISP can silently update the firmware of the wireless AP for all hotspots in the network. The maps of these hotspot networks are available on-line, integrated in Google Maps, on dedicated website [16, 26]. By inspecting the traffic generated when visiting the map websites, we were able to extract the url to obtain the list of hotspots (together with their GPS coordinates) located in a given geographic region described by its north east and south west corner. Because the number of returned hotspots was limited to a hundred, we recursively split the region of interest in four until all the hotspots were returned. For the Paris area, the average density is $564 \pm 270 \mathrm{APs} / \mathrm{km}^{2}$ (resp. $853 \pm 346 \mathrm{APs} / \mathrm{km}^{2}$ ), and goes up to $1450 \mathrm{APs} / \mathrm{km}^{2}$ (resp. $1560 \mathrm{APs} / \mathrm{km}^{2}$ ) in populated areas for Free (resp. SFR). The FON [14] hotspots network has an even higher density as it includes the SFRWifi network. For comparison, the adversarial network of sniffing APs considered in our study has a density of $1138 \mathrm{APs} / \mathrm{km}^{2}$ (37 APs deployed over a region of size $130 \mathrm{~m} \times 250 \mathrm{~m}$ ), which is even lower than that of the real networks in densely populated areas. Another illustrative example is that of a company exploiting its network of $\mathrm{WiFi}$ stations to infer the social relationships between its employees. For instance, EPFL has $~ 880 \mathrm{WiFi}$ APs deployed over a region of $500 \mathrm{~m} \times 800 \mathrm{~m}$, that is a density of $2200 \mathrm{APs} / \mathrm{km}^{2}$. As these statistics suggest, there exist examples of deployed WiFi AP networks that have a similar or higher density than the adversary considered in this paper, which shows that the results presented in our work are realistic and applicable to existing networks.

\section{RELATED WORK}

Eagle et al. [11,12] studied complex social systems by collecting Bluetooth encounter data from 94 students equipped with mobile phones. In particular, they identified proximity patterns between devices and performed relationship inference on the data by comparing the results with the ground truth obtained from surveys and answers to questionnaires. Similarly to other user studies [10,23], the authors could only compare their findings (based on Bluetooth encounters between devices) with the ground truth, without having access to a network infrastructure that would allow them a more thorough analysis by comparing these results with those obtained by a passive third-party adversary. However, off-campus location data was collected in $[11,12]$ and it significantly improved the quality of the inference results. A recent study [3], performed on a group of 27 participants, shows that it is possible to accurately infer the social ties between people based on Bluetooth encounters, phone calls, SMSs and cell-tower IDs, without any prior knowledge about the participants. Nevertheless, these findings are based on data that is available only on the local devices, therefore it is unclear to what extent the results apply to the inference success of an external adversary. Other studies focused on inferring the social ties from co-presence of users, as in the case of Flickr [9], and on dynamics of inter-encounter times [28]. A large user study, involving 168 participants over 2 years, was conducted in [20] in order to study people's behavior with respect to mobile phone usage, activity and location.With regard to location privacy in pervasive wireless networks, [6] studied the efficacy of dynamic allocation of pseudo-random IDs in specific regions called mix-zones [4], showing that in deployed networks such a mechanism provides only limited success in protecting users' location privacy. 


\section{CONCLUSION AND FUTURE WORK}

In this work, we experimentally evaluate a practical adversary's accuracy in inferring the type of relationship of users in ubiquitous networks. The availability of source-diversity in our experiment, enables us to compare the accuracy of an external adversary with that of an internal adversary (e.g., a malicious application) who has access to actual proximity information stored on the devices. Our results show the following two aspects. First, social ties are exposed to a significant inference threat by an external adversary, who can quite accurately infer the type of social ties between users simply by relying on location estimates and encounter characteristics, compared to a curious application running on the device (or to the experimenter). Second, by applying a well-suited community detection algorithm, the adversary can double his inference accuracy on the same data set.

As part of our future work we intend to evaluate the efficacy of existing countermeasures as well as design a scheme that would limit the adversary's accuracy, for example by dynamically changing the fixed identifiers (MAC addresses) based on time- and location-context. We also intend to study the effect of the density of the WiFi APs and consider additional features such as the time at which the encounters occur. Finally, we plan to extend our experiments offcampus and refine the relationship categories accordingly.

\section{ACKNOWLEDGEMENTS}

This work has been partially conducted while F. Lopez was with EPFL and P. Ginzboorg and V. Niemi were with Nokia. We would like to thank Imad Aad for the fruitfull discussions, as well as Nokia for supporting this project.

\section{REFERENCES}

[1] I. Aad, M. Jadliwala, I. Bilogrevic, V. Niemi, J. Hubaux, P. Ginzboorg, and K. Leppänen. Nokia Instant Community at EPFL: A Real-World Large-Scale Wireless Peer-to-Peer Trial. Technical Report 170421, EPFL, 2011.

[2] H. Akaike. A New Look at the Statistical Model Identification. IEEE TAC, 19:716-723, 1974.

[3] J. Banford and J. Irvine. Estimating social graphs in an education environment. IEEE VT Mag., 7(1), 2012.

[4] A. Beresford and F. Stajano. Location Privacy in Pervasive Computing. IEEE Perv. Comp., 2, 2003.

[5] I. Bilogrevic, M. Jadliwala, I. Lám, I. Aad, P. Ginzboorg, V. Niemi, L. Bindschaedler, and J. Hubaux. Big brother knows your friends: on privacy of social communities in pervasive networks. Pervasive, pages 370-387, 2012.

[6] L. Bindschaedler, M. Jadliwala, I. Bilogrevic, I. Aad, P. Ginzboorg, V. Niemi, and J. Hubaux. Track me if you can: On the effectiveness of context-based identifier changes in deployed mobile networks. In NDSS, 2012.

[7] S. P. Borgatti, A. Mehra, D. J. Brass, and G. Labianca. Network Analysis in the Social Sciences. Science, 323:892-895, 2009.

[8] A. Bose and C. Foh. A Practical Path Loss Model for Indoor WiFi Positioning Enhancement. In ICICS, 2007.

[9] D. Crandall, L. Backstrom, D. Cosley, S. Suri, D. Huttenlocher, and J. Kleinberg. Inferring Social
Ties from Geographic Coincidences. Proc. Nat. Ac. Sc., 107:22436-22441, 2010.

[10] C. Diot. Haggle Project. http://www.haggleproject.org/. Last visited 13/Mar/2013.

[11] N. Eagle and A. Pentland. Reality mining: sensing complex social systems. Pers. Ubiq. Comp., 10:255-268, 2006.

[12] N. Eagle, A. Pentland, and D. Lazer. Inferring Friendship Network Structure by Using Mobile Phone Data. Proc. Nat. Ac. Sc., 106:15274-15278, 2009.

[13] Femto Forum. http://femtoforum.org/fem2/pressreleases.php ?id=277, Last visited 13/Mar/2013.

[14] FON. http://corp.fon.com/en. Last visited 13/Mar/2013.

[15] S. Fortunato. Community Detection in Graphs. Physics Reports, 486, 2010.

[16] Map of FreeWifi Hotspots. http://iphone.rOro.fr/fwConnect/map.php, Visited on $01 /$ Nov/2012.

[17] N. Gong, M. Laibowitz, and J. Paradiso. Dynamic Privacy Management in Pervasive Sensor Networks. In Proc. Int. Joint Conf. on Ambient Intell., 2010.

[18] B. Hoh, M. Gruteser, H. Xiong, and A. Alrabady. Enhancing Security and Privacy in Traffic-Monitoring Systems. IEEE Perv. Comp., 5:38-46, 2006.

[19] H. Hu, S. Myers, V. Colizza, and A. Vespignani. Wifi networks and malware epidemiology. Proc. Nat. Ac. Sc., 106(5):1318, 2009.

[20] N. Kiukkonen, J. Blom, O. Dousse, D. Gatica-Perez, and J. Laurila. Towards Rich Mobile Phone Datasets: Lausanne Data Collection Campaign. In ICPS, 2010.

[21] Y. Matsuo, N. Okazaki, K. Izumi, Y. Nakamura, T. Nishimura, and K. Hasida. Inferring Long-term User Property based on Users. In IJCAI, 2007.

[22] A Definitive List of NFC Phones. http://www.nfcworld.com/nfc-phones-list/, Last visited 13/Mar/2013.

[23] T. Nicolai, E. Yoneki, N. Behrens, and H. Kenn. Exploring Social Context with the Wireless Rope. In OTM, 2006.

[24] G. Palla, I. Derényi, I. Farkas, and T. Vicsek. Uncovering the Overlapping Community Structure of Complex Networks in Nature and Society. Nature, $435,2005$.

[25] Qualcomm AllJoin. https://developer .qualcomm.com/develop/ mobile-technologies/peer-peer-alljoyn, Last visited 13/Mar/2013.

[26] SFR Carte Wifi. http://cartewifi.sfr.fr, Last visited 13/Mar/2013.

[27] P. Traynor, K. Butler, W. Enck, P. McDaniel, and K. Borders. Malnets: Large-Scale Malicious Networks via Compromised Wireless Access Points. Security and Communication Networks, 3(2):102-113, 2010.

[28] E. Yoneki, D. Greenfield, and J. Crowcroft. Dynamics of Inter-Meeting Time in Human Contact Networks. In Proc. Int. Conf. on Adv. in Soc. Net. Analysis and Mining, 2009. 